\title{
"Mais qui ose en affronter les suites ? " : Emil Cioran et les rapports entre la vanité, le suicide et l'écriture
}

Vers le début du Précis de décomposition, Emil Cioran expose le problème de la vanité en deux questions très simples : "Qui n'est pas imbu de la conviction que tout est vain ? Mais qui ose en affronter les suites? " (2011, p. 45). En quoi lécrivain cioranien s'associe-t-il à cette humanité généralisée et en quoi est-ce qu'il s'en distingue ? Cioran s'invente une voix qui surgit des ruines de la Deuxième Guerre Mondiale. Le Précis, son premier livre publié après la guerre, est aussi son premier livre en français, une voix qui émerge ex nihilo pour remettre l'accent sur la tension entre la vanité et l'espoir auquel nous nous obstinons à croire malgré nous ${ }^{1}$.

Quelle doit être notre réaction alors, face à la vanité de tout ? On pourrait être tenté de dire que la destruction, et surtout l'autodestruction, semble être la solution la plus raisonnée. Bien que Cioran écrive de manière quasi-obsessionnelle sur le thème du suicide, il n'arrive pas à le recommander comme solution à la vanité justement parce que la vanité résiste à toute solution. L'écrivain se voit ainsi dans une position contradictoire, car à quoi sert-il de déclarer la vanité de tout si cette déclaration ne fait que participer à cette vanité ? Publier et lire des livres pourraient-ils faire plus que de nous rendre simplement conscients de la vanité de tout? En quoi l'écriture

- Joseph Acquisto - professeur titulaire de littérature française à l'Université du Vermont (USA). Adresse pour correspondance : 517 Waterman Bldg, University of Vermont, Burlington, VT, 05405, USA ; e-mail : jacquist@uvm.edu

1. En présentant Le Précis, Maurice Nadeau donne cette description de la voix qui y parle : «Ce n’est ni un monstre, ni un phénomène, à peine un provocateur et peut-être pas du tout un "suicidé » en sursis, mais c'est sûrement un être submergé de honte, de dégoût et qui souffre. Sur le bord de la voie triomphale qui mène à la destruction atomique il pose le sac et s'assied, fourbu, refusant toutes les consolations et toutes les pitiés » $(2009$, p. 212). 
et la lecture en tant que création et destruction simultanées nous permettent-elles d'oublier la vanité au moins assez pour continuer à vivre?

Dans la contradiction impliquée par les questions de Cioran s'annonce tout un programme que son œuvre entière tente d'affronter. Il continue en suggérant que

l'homme à vocation métaphysique est plus rare qu'un monstre - et pourtant chaque homme contient virtuellement les éléments de cette vocation. Il a suffi à un prince hindou de voir un infirme, un vieillard et un mort pour tout comprendre; nous qui les voyons, nous ne comprenons rien, car rien ne change dans notre vie. Nous ne pouvons renoncer à quoi que ce soit ; cependant les évidences de la vanité sont à notre portée. Malades d’espoir, nous attendons toujours; et la vie n'est que l'attente devenue hypostase. Nous attendons tout - même le Rien plutôt que d'être réduits à une suspension éternelle [...]. [...] L'humanité vit amoureusement dans les événements qui la nient... (2011, p. 45)

L'impossibilité de vivre les conséquences de l'assentiment intellectuel à la vanité de tout évoque une fissure ou une division au cœur du moi où la lucidité ne saurait aller de pair avec l'expérience vécue quotidienne ${ }^{2}$. Dès l'abord de la question de la vanité, il paraît que la seule solution, la seule manière de tirer les conséquences, d' " en affronter les suites », serait le suicide qui mettrait fin à cette maladie provoquée (ou manifestée) par l'impossibilité de renoncer à l'espoir.

$\mathrm{La}$ « suspension éternelle ${ }^{3}$ » se manifeste par l'action de tourner en rond, puisque nous sommes incapables du mouvement mais, en même temps, nous ne saurions nous tenir tranquilles. Un peu plus loin, dans le Précis, il revient à cette idée :

Se répéter à soi-même mille fois par jour : « Rien n’a de prix ici-bas », se retrouver éternellement au même point, et tournoyer niaisement comme une toupie... Car il n'y a pas de progression dans l'idée de la vanité du tout, ni d'aboutissement ; et, aussi loin que nous nous hasardions dans cette rumination, notre connaissance ne s'accroît aucunement [...]. C'est un arrêt dans l'incurable, une lèpre de l'esprit, une révélation par la stupeur. Un simple d'esprit, un idiot, qui subirait une illumination, et qui s'y installerait sans aucun moyen d'en sortir et de recouvrer sa condition nébuleuse et confortable, tel est l'état de celui qui se voit engagé malgré lui dans la perception de l'universelle futilité. [...] Comme il est révolu le chaos, reposant et calme, d'avant la terrible Création, ou, plus doux encore, le chaos du néant mental! (2011, p. 87-88)

2. Ailleurs Cioran situe cette division du moi au cœur de la philosophie moderne : « La philosophie moderne, en instaurant la superstition du Moi, en a fait le ressort de nos drames et le pivot de nos inquiétudes. [...] [N]ous nous sommes voulus sujets, et tout sujet est rupture avec la quiétude de l'Unité. [...] Nous mesurons la valeur de l'individu à la somme de ses désaccords avec les choses, son incapacité d'être indifférent, à son refus de tendre vers l'objet » (2011, p. 273).

3. Je développe plus longuement les conséquences de la suspension entre l'espoir et le désespoir dans le cadre d'une discussion de l'impossibilité du néant et du renoncement au salut de la part de Cioran dans The Fall out of Redemption (Acquisto, 2015, p. 119-69). 
L'infinitif en tête de ce passage souligne l'impossibilité de la progression temporelle et marque l'absence d'une subjectivité précise et d'une temporalité. Celui qui était «l'homme à vocation métaphysique » dans le premier passage se voit réduit (ou élevé) au rang d'un "simple d'esprit ». Une fois que nous passons de l'intemporalité de l'infinitif à l'hypothétique du conditionnel (ce sujet « subirait une illumination »), force nous est de mettre en question l'universalité du sujet, étant donné que le sujet en question est maintenant celui qui serait un illuminé, ce qui établit une division entre ceux qui ont subi l'illumination et ceux qui ne l'ont pas fait. De plus, ce conditionnel met en question la possibilité même de cette illumination. Du coup, ce qui sétait annoncé comme universel et nécessaire semble réduit à la particularité.

Ce qui semblait être une question philosophique, celle de l'impossibilité de vivre en toute connaissance de la vanité de l'action, devient une question littéraire, car elle est intimement liée à la question de qui parle dans le texte. L'écrivain s'inclut-il dans les rangs de l'humanité en général ? Se compte-t-il parmi les illuminés ? En tire-t-il un sens de supériorité ? Si tel est le cas, il ne saurait plus se compter parmi l'humanité en général à cause de l'illumination réservée à certains. Ou peut-être ce discours universalisant ne fait-il que masquer un échec personnel sous la guise des déclarations générales. La section intitulée « Effigie du raté » complique encore ces questions :

Ayant tout acte en horreur, il se répète à lui-même : «Le mouvement, quelle sottise !» Ce ne sont pas tant les événements qui l'irritent que l'idée d'y prendre part; et il ne s'agite que pour s'en détourner. [...] C'est un Ecclésiaste de carrefour, qui puise dans l'universelle insignifiance une excuse à ses défaites. Soucieux de trouver sans importance quoi que ce soit, il y réussit aisément, les évidences étant en foule de son côté. Dans la bataille des arguments, il est toujours vainqueur, comme il est toujours vaincu dans l'action : il a « raison ». Il rejette tout - et tout le rejette. Il a compris prématurément ce qu'il ne faut pas comprendre pour vivre - et comme son talent était trop éclairé sur ses propres fonctions, il l'a gaspillé de peur qu'il ne s'écoulât dans la niaiserie d'une œuvre. Portant l'image de ce qu'il eût pu être comme un stigmate et comme un nimbe, il rougit et se flatte de l'excellence de sa stérilité [...]. Il extrait sa liberté de l'immensité de ses inaccomplissements ; c'est un dieu infini et pitoyable qu'aucune créature n'adore, et que personne n'épargne. Le mépris qu'il a déversé sur les autres, les autres le lui rendent. Il n'expie que les actes qu'il n'a pas effectués, dont pourtant le nombre excède le calcul de son orgueil meurtri. Mais à la fin, en guise de consolation, et au bout d'une vie sans titres, il porte son inutilité comme une couronne. (2011, p. 81)

Le fait que cette affirmation de la vanité arrive, dans "Effigie du raté ", dans le contexte d'une caractérisation de l'homme raté plutôt que de l'homme universel impose des questions d'identité essentielles. Sommes-nous tous des ratés ? Ou y a-t-il une division entre ceux qui sont des ratés et ceux qui ne le sont pas ? La question de l'identité de la voix narrative revient : Cioran se compte-t-il parmi les ratés ou se tient-il à l'écart pour les juger ? S'agit-il d'une auto-condamnation 
à la troisième personne ? Si oui, est-ce possible de reconnaitre la vanité de tout sans devenir un «Ecclésiaste de carrefour » ? Ou l'évocation de la vanité ne sert-elle peut-être que d' « excuse » de la part de Cioran comme de la part du raté. L'équivoque du passage est presque total, car des " inaccomplissements » du raté vient sa " liberté », tandis que le mépris mutuel invoqué vers la fin du passage suggère qu'il s'agit de la rancune.

Est-il donc vain d'invoquer la vanité ? Ne serait-ce qu'une tentative de cacher son propre échec en essayant de l'assimiler à un échec généralisé ? Ou bien l'échec personnel révèle-t-il quelque chose d'essentiel à propos de l'impossibilité de « réussir » en général ? Aux yeux de qui le raté a-t-il échoué ? Dans le second paragraphe de la section, le « il » se convertit en «nous » en passant par le « on » :

("À quoi bon ? » - adage du Raté, d'un complaisant de la mort. Quel stimulant lorsqu'on commence à en subir la hantise ! Car la mort, avant de trop nous y appesantir, nous enrichit, nos forces s'accroissent à son contact ; puis, elle exerce sur nous son œuvre de destruction. L'évidence de l'inutilité de tout effort, et cette sensation de cadavre futur sérigeant déjà dans le présent, et emplissant l'horizon du temps, finissent par engourdir nos idées, nos espoirs et nos muscles, de sorte que le surcroît délan suscité par la toute récente obsession, - se convertit - lorsque celle-ci s’est implantée irrévocablement dans l'esprit - en une stagnation de notre vitalité. Ainsi cette obsession nous incite à devenir tout et rien. [...] [Q]uand, harcelés au milieu de notre vie, nous sommes aussi loin du ciel que de la vulgarité, elle nous transforme en cette espèce de héros décomposés qui promettent tout et n'accomplissent rien : oisifs s'essoufflant dans le Vide ; charognes verticales, dont la seule activité se réduit à penser qu'ils cesseront d'être...) (2011, p. 81-82)

Que reste-t-il à dire après l'affirmation de l'à-quoi-bon ? La question est plus complexe qu'elle ne semble au premier abord à cause de l'écart entre la connaissance de la vanité et les conséquences à en tirer. Doit-on s'abandonner à la lamentation ? Passer au suicide ? Ces deux options semblent dépourvues d'intérêt face à une vanité qui les engloberait. Cioran s'enfonce-t-il encore plus loin dans la vanité en la développant comme thème? Y trouve-t-il une issue ? Et jusqu'à quel point est-ce que la lucidité compte comme marque de supériorité ou d'infériorité ? Si le moi qui se révèle ici s'avère fissuré ou contradictoire, la réponse du lecteur doit l'être aussi, ce qui établit un rapport de conspiration entre le texte et son lecteur.

Nicolas Cavaillès (2011) a identifié deux voix distinctes dans l'écriture cioranienne, la «suicidaire » et la « décomposée »; celle-ci s’associe surtout aux œuvres françaises :

Si les thèmes qui la [la voix décomposée] préoccupent sont semblables, c'est avec scepticisme, finesse et érudition, qu'ils sont abordés. La vanité du monde, des espoirs et des idéologies, est exposée sans étonnement [...]. [...] Un cynisme froid y croise une sentimentalité douloureuse, tournée vers ses erreurs et vers sa naïveté d'antan ; Cioran s'isole, à distance des autres, de son siècle et de lui-même, dans une hyper-conscience de soi qui n'exclut pas, in fine, de douces larmes mélancoliques ni un fin sourire moqueur. (p. 97) 
Reste à déterminer si cette voix plus calme, plus semblable à celle de l'Ecclésiaste réussit à dominer dans l'écriture cioranienne à propos de la vanité ou s'il s'agit de ce que Cavaillès identifie comme la "voix corrompue », un mélange des deux autres (2011, p. 97). Dans le cas du traitement de la vanité, il est peut-être moins question d'un mélange que d'une tension à l'intérieur de la question. À quoi bon râler contre la vanité si l’on sait à l'avance que ce râlement est incapable de l'annuler ? La voix cioranienne affirme l'écriture tout en vantant la supériorité du silence : « Tout mot est un mot de trop. Il s'agit pourtant d'écrire : écrivons... dupons-nous les uns les autres » (Cioran, 2011, p. 333) ${ }^{4}$. Comme l'a noté Cavaillès (2011), "Cioran écrit le Précis dans la corruption de ses instincts suicidaires et de son raffinement décomposé - tel un homme qui, sautant d'un pont, proclamerait, placide et provocateur, qu'il est vain de sauter d'un pont» (p. 97).

Étant donné toutes ces tensions et contradictions et l'impossibilité de se prononcer définitivement sur le rapport entre la voix cioranienne et le Raté, comment lire Cioran à propos de la vanité ? À certains moments, la réponse à la vanité s'accompagne d'une pointe d'humour qui crée un certain détachement par rapport à la tragédie de la situation : "Ce matin, après avoir entendu un astronome parler de milliards de soleils, j'ai renoncé à faire ma toilette : à quoi bon se laver encore? » (2011, p. 1027). Ailleurs, Cioran affirme que l'aveu de la vanité apporte une espèce de plaisir :

Il existe un indéniable plaisir à savoir que tout ce qu’on fait n'a aucune base réelle, que c'est tout un de commettre un acte ou de ne pas le commettre. Il n'en demeure pas moins que dans nos gestes quotidiens nous composons avec la Vacuité, c'est-à-dire que, tour à tour et parfois en même temps, nous tenons ce monde pour réel et irréel. Nous mélangeons là vérités pures et vérités sordides, et cette mixture, honte du penseur, est la revanche du vivant. (2011, p. 1042)

Le lecteur de Cioran demeure avec lui dans l'incertitude, vacillant entre le rire et les larmes dans l'impossibilité de se prononcer définitivement.

Il sied alors de considérer les conséquences potentielles de l'affirmation de la vanité chez Cioran. Avant tout, la conscience de la vanité risque d'annuler tout désir d'agir :

Savoir que rien ne vaut la peine devient implicitement une croyance, donc une possibilité d'acte ; c'est que même un rien d'existence présuppose une foi inavouée ; un simple

4. Voir à ce propos les remarques de Claude Mauriac : "Cioran ne se pardonne pas de continuer à écrire. Seul le silence est grand. Il est de la race de ceux qui ne l'ignorant point, ne peuvent pour autant renoncer à la parole, à l'écriture surtout. Cioran le désespéré est un homme de lettres. Contradiction à laquelle il est sensible. Occasion nouvelle de se moquer de lui-même avec un ricanement non tout à fait dépourvu de complaisance » (2009, p. 215). 
pas - fût-il vers un semblant de réalité - est une apostasie à l’égard du néant ; la respiration elle-même procède d'un fanatisme en germe, comme toute participation au mouvement... Depuis la flânerie jusqu'au carnage, l'homme ne parcourt la gamme des actes que parce qu'il n'en perçoit point le non-sens [...]. (2011, p. 69)

Pour fonctionner et vivre de jour en jour, il faut oublier ce que nous savons à propos de la vanité et vivre comme si tout avait un sens, comme si on ne savait pas que tout est vain. Cela ne fait que renforcer la division du moi puisque le moi qui pense et le moi qui agit ne peuvent pas coexister, étant donné que l'un annule l'autre. Dans ce scénario, on ne tire pas les conséquences de la vanité ; on les oublie tout court.

Ce n'est pas à dire qu'agir est toujours préférable et que nous perdons quelque chose de valable en abandonnant l'action. Le moi qui agit chez Cioran est capable de toute la gamme d'émotions fortes, y inclus la haine. Se rendre compte de la vanité en refusant l'action élimine une haine que l'on n'est plus capable de sentir à l'égard des individus, parce que la haine est généralisable face à l'horreur qu'est le monde :

Je ne hais personne ; - mais la haine noircit mon sang et brûle cette peau que les années furent incapables de tanner. [...] J'ai voulu aimer la terre et le ciel, leurs exploits et leurs fièvres, - et n'y ai rien trouvé qui ne me rappelât la mort : fleurs astres, visages, - symboles de flétrissure, dalles virtuelles de toutes les tombes possibles ! [...] Ceux qui croient à leur vérité [...] laissent après eux le sol parsemé de cadavres. [...] Qu'on me montre ici-bas une seule chose qui a commencé bien et qui n'a pas fini mal. [...] Dans ces conditions, sur qui déverser sa haine? Nul n'est responsable d'être, et encore moins d'être ce qu'il est. [...] C’est ainsi que dans un monde où tout est haïssable, la haine devient plus vaste que le monde, et, pour avoir dépassé son objet, s’annule. (2011, p. 73)

Si l'existence entière est digne d'une haine que nous ne sommes pas capables de ressentir parce qu'elle s'annule, reste la possibilité d’en finir avec tout.

Face à la haine du monde et à la vanité de l'existence, le suicide serait-il alors recommandé ? Cioran en parle inlassablement. À première vue, mettre fin à une existence vaine semble une option efficace et raisonnable. Et pourtant, rien n'est simple dans l'univers cioranien :

La consolation par le suicide possible élargit en espace infini cette demeure où nous étouffons. L'idée de nous détruire, la multiplicité des moyens d'y parvenir, leur facilité et leur proximité nous réjouissent et nous effraient ; car il n'y a rien de plus simple et de plus terrible que l'acte par lequel nous décidons irrévocablement de nous-mêmes. En un seul instant nous supprimons tous les instants ; Dieu lui-même ne saurait le faire. Mais, démons fanfarons, nous différons notre fin : comment renoncerions-nous au déploiement de notre liberté, au jeu de notre superbe? (2011, p. 36) 
La solution suicidaire est presque toujours et simultanément accessible et inaccessible pour la plupart d'entre nous. Et l'impossibilité de passer à l'acte risque de nous rendre obsessionnels. Dans ce qui pourrait être une autocritique ou une affirmation d'une impossibilité nécessaire, Cioran constate que «l'obsession du suicide est le propre de celui qui ne peut ni vivre ni mourir, et dont l'attention ne s'écarte jamais de cette double impossibilité "(2011, p. 667). Ici encore il y a quelque chose qui bloque la possibilité d' "affronter les suites ", ce qui ne fait que renforcer la futilité de méditer sur la vanité de tout si nous sommes incapables d'y réagir. Un parallèle s'annonce entre le suicide et la futilité dans le sens qu'il n'y a rien de plus évident que ces deux phénomènes et pourtant rien de plus difficile à reconnaître pleinement : "Or, - pourquoi se le dissimuler ? - la futilité est la chose du monde la plus difficile, j’entends la futilité consciente, acquise, volontaire " (2011, p. 338). Le suicide implique un certain héroïsme : «Point d'élus, me répétais-je, en dehors de ceux qui se donnent la mort. Maintenant encore, j'estime plus un concierge qui se pend qu'un poète vivant» (2011, p. 152). Et le fait de continuer à vivre ne va pas sans une certaine honte: "Toutes nos humiliations viennent de ce que nous ne pouvons pas nous résoudre à mourir de faim » (2011, p. 153).

Ce qui empêche le suicide de servir comme remède à la vanité et à la futilité, c'est qu'en fin de compte, il n'arrive pas à l'annuler. C'est la vanité qui aura le dernier mot : « Ce n'est pas la peine de se tuer, puisqu'on se tue toujours trop tard » (2011, p. 756). L'idée centrale chez Cioran de " penser contre soi » (c'est le titre de la première section de La Tentation d'exister) l'empêche de se prononcer à propos du suicide comme solution à quoi que ce soit. Les textes de Cioran ne peuvent pas sortir de la contradiction pour la résoudre ; tout effort pour arriver à une solution serait vain. Une fois que nous reconnaissons la vanité, nous sommes pour ainsi dire déjà morts. Cette mort métaphorique rendrait ridicule le suicide. Dans un passage adressé à un «tu » non-identifié (une voix qui se parle ou qui s'adresse au lecteur ?), l'auteur évoque l'effet néfaste et, d'une manière figurée au moins, mortifiante de la futilité :

Pourquoi n'as-tu la force de te soustraire à l'obligation de respirer ? Pourquoi subir encore cet air solidifié qui bloque tes poumons et sécrase contre ta chair ? [...]

Ouvrir tes veines pour inonder cette feuille qui t'irrite comme t'irritent les saisons ! Ridicule tentative! Ton sang, décoloré par les nuits blanches, a suspendu son cours... Rien ne réveillera en toi la soif de vivre et de mourir, éteinte par les années, à jamais rebutée par ces sources sans murmure ni prestige auxquelles s'abreuvent les hommes. (2011, p. 86)

Tout mène à croire alors que le suicide, bien qu'il soit une tentation chez Cioran, tombe lui aussi victime de la vanité plutôt que de servir de remède 5 .

5. Dans un entretien, Cioran maintient une séparation entre l'idée du suicide, qui représente pour lui la liberté et le soulagement, et le passage à l'acte : « Je n'ai jamais dit qu'il fallait se suicider ; j’ai simplement 
Reste alors l'écart entre savoir et vivre, ce que nous savons (ou ce que nous avons la possibilité de savoir) à propos de la vanité de tout contre l'action que la vie quotidienne nous impose. Si la vanité paraît évidente à maintes reprises dans l'ouvre de Cioran, à d'autres moments il indique qu'un effort est nécessaire pour s'en convaincre ou même s'en rendre compte. Dans son dernier livre, nous trouvons cet aphorisme : "Dévorer biographie après biographie pour mieux se persuader de l'à quoi bon de n'importe quelle entreprise, de n'importe quelle destinée " (2011, p. 1090). Une fois la connaissance de la futilité acquise, rien de plus impossible que d'en accepter les conséquences pleinement : «Personne n'a été autant que moi persuadé de la futilité de tout, personne non plus n'aura pris au tragique un si grand nombre de choses futiles » (2011, p. 828). Comme dans le cas du suicide, il est difficile d'aller jusqu'au bout, et dans le cas de la futilité, il n'est pas bien clair à quoi servirait une vie où l'on se rendait pleinement compte des conséquences de la futilité de toute façon. À certains moments pourtant, Cioran est prêt à reconnaître un aspect salvifique dans la conscience de la vanité :

Rien n'importe : grande découverte s'il en fut, et dont personne n'a su tirer profit. À cette découverte, réputée déprimante, le vide seul, dont elle est la devise, peut donner un tour exaltant, lui seul s'emploie à convertir le négatif en positif, l'irréparable en possible. Qu'il n'y ait pas de soi, nous le savons mais c'est un savoir grevé d'arrière-pensées. Le vide est heureusement là, et quand le soi s'efface, il en tient lieu, il tient lieu de tout, il comble nos attentes, il nous apporte la certitude de notre non-réalité. Le vide, c'est l'abîme sans vertige. (2011, p. 681)

Habiter l'abîme n'est pourtant pas à notre portée ; tôt ou tard, les tensions et contradictions de l'expérience vécue nous ramènent sur la route de la tension inexorable.

Quel est donc le rôle de l'écrivain face à la vanité ? Il peut la déclarer, la décrire, la dénoncer, la louer, mais finalement l'acte d'écrire, comme le suicide ou n'importe quelle autre action, ne fait qu'ajouter à la vanité et à la futilité de tout. En ce sens, l'auteur ne se tient pas à l'écart de ce nous par lequel il désigne l'humanité. Comme la voix cioranienne, que l'on pourrait considérer comme un "personnage » qui parle à l'intérieur d'une situation établie par le texte ${ }^{6}$, l'auteur lui-même, celui qui crée la voix

dit que seule l'idée du suicide pouvait nous aider à supporter la vie. L'idée qu'il soit à portée de notre main de mettre fin à nos jours, que nous puissions à tout moment nous suicider si nous le voulons peut représenter un énorme soulagement. Du moins cette perspective m’a-t-elle personnellement beaucoup aidé et j'ai exposé ce raisonnement à tous ceux qui m’ont déclaré vouloir se tuer » (Liiceanu, 1995, p. 134-5).

6. Voir ces remarques d'Ilinca Zarifopol-Johnston à propos du Cioran de Sur les cimes du désespoir, son premier livre, que nous pourrions appliquer au moi textuel cioranien de son œuvre en général : « le narrateur est un suicidé, un auto-tueur. Mais pour Cioran, ce moi fictif est un geste rhétorique et théâtral par lequel il aspire à sauver son propre moi. En se mettant dans le rôle de ce personnage, Cioran se suicide de manière métaphorique tout en arrivant à survivre à l'appel à la mort en éliminant, à travers son personnage inventé, le surplus d'énergie lyrique qu'il ressentait monter en lui » (2009, p. 77 ; c'est moi qui traduis). 
et la situation, se trouve à la fois au-dessus de l'humanité qu'il analyse et tout à fait ancré dedans. Comme le remarque Sylvain David : « Assez ironiquement, c'est justement en tenant de se fonder comme être " autonome » que - selon sa propre vision du monde - Cioran s'inscrit comme "fait social" " (2006, p. 11). Dans ces textes dépourvus de toute allusion aux autres penseurs, Cioran essaie de s'isoler non seulement de l'humanité en général mais de la communauté des penseurs et des écrivains en particulier pour créer l'illusion d'une voix solitaire émergeant du néant :

Cioran reprend à sa manière cette idée d'un sacrifice personnel, d'un exploit vain, mais à un niveau strictement intellectuel : [...] il aspire moins à se détruire physiquement qu'à démolir systématiquement en lui la moindre idée, image ou illusion qu'il pourrait avoir en commun avec ses contemporains. De son point de vue, l'unique exploit, le seul geste héroïque possible encore à l'homme moderne est celui, exemplaire, de "penser contre soi ». (David, 2006, p. 15)

Dans un des rares instants où Cioran se sert du mot «vanité » dans le sens d'orgueil plutôt que de futilité, il se voit comme presque divin par sa capacité de se détacher de tout : «J'essaie - sans succès - de ne plus tirer vanité de rien. Quand j'y arrive pourtant, je sens que je n'appartiens plus au gang des mortels. Je suis alors au-dessus de tout, des dieux eux-mêmes. C'est peut-être cela la mort : une sensation de grande, d'extrême supériorité » (2011, p. 666). La difficulté que la voix cioranienne indique souvent - quant à la possibilité de vivre selon ses propres recommandations - suggère que l'écrivain n'est presque jamais au-dessus des autres et que la sagesse qu'il prétend avoir acquis ne sert pas à altérer son vécu ${ }^{7}$. Plus cette voix tente de se séparer et de s'isoler des autres, plus elle est ramenée dans le cadre de l'expérience humaine dont elle aurait voulu s'échapper. La voix venant de nulle part reste la voix de chacun, d'où la nécessité de renouveler l'acte de penser contre soi.

À quoi bon écrire alors? Cioran se pose la question dans ses Cahiers :

Je ne crois pas aux livres, j'entends aux livres à publier. Celui que j'ai fini $[\ldots]$ attend là et je ne peux me décider à le porter chez l'éditeur. C'est que je ne crois pas utile de publier un autre livre. À quoi bon ? Un livre est un acte de naïveté. Or j'ai la prétention d'être détrompé au-delà du permis. (1997, p. 564)

Ailleurs, il imagine l'acte d'écrire comme une agression : « [Q]u'est-ce qu'un livre sinon une perte d'innocence, un acte d'agression, une répétition de notre chute?

7. Voir l'article de Mihaela-Gentiana Stanisor (2015) qui remarque, à propos du moi cioranien : «Le sujet est l'égo cogito cartésien qui prend le poids du moi empirique pour arriver au « je » transcendantal, ce « je » en relation avec un autre ou les autres. Cioran écrit et s'écrit. Sa démarche, qui le tient toujours près du mot, vise l'assurance d'être soi-même agissant et souffrant » (p. 52). Selon Stanisor, les écrits de Cioran sont "moitié confession, moitié (con)damnation" (2015, p. 52). 
Publier ses tares pour amuser ou exaspérer ! Une barbarie à l'égard de notre intimité, une profanation, une souillure. Et une tentation » (2011, p. 332). Il serait mieux, d'après ce commentaire, de garder le silence mais l'écrivain ne réussit pas à éviter la tentation de publier. Ainsi Cioran établit un parallèle entre l'écriture et tout autre acte : l'on fait tout en dépit de ce que l'on sait à propos de la vanité et les avantages qu'il y aurait à tout cesser au nom de cette vanité. Mais l'inaction et le silence s'avèrent impossibles, la tentation de vivre et d'écrire étant trop forte malgré tout. L'écriture exige l'illusion et nous entraîne plus loin dans cette illusion :

La stérilité rend lucide et impitoyable. Dès qu'on cesse de produire, on trouve sans inspiration et sans substance tout ce que font les autres. Jugement sans doute vrai. Mais il fallait le porter avant, lorsqu'on produisait, lorsque justement on faisait comme les autres. (2011, p. 948)

La révélation de la vanité vient toujours trop tard face à la tentation de vivre. Et ici il y a quand même une sorte d'affirmation de la vie sous la guise de l'écriture qui est condamnable mais qui demeure comme trace de vie et d'action, dans le sens où Cioran se sert de l'écriture pour râler contre la vanité et en même temps affirmer la vanité de râler contre elle. L'écriture est à la fois un acte d'oubli et de rappel de la vanité puisque l'écrivain l'oublie pour mieux s'en souvenir.

En fin de compte, l'écrivain ne se sépare plus des autres. Ce qu'il ressent à propos de l'écriture se lie à tout autre genre d'activité et ramène l'écrivain dans le domaine du vécu malgré tout ce qu'il sait au sujet de la vanité. Celle-ci devient alors une force négatrice si puissante qu'elle permet et encourage même la négation de sa propre négation $^{8}$. Elle joue un rôle ironique. Mircea Eliade analyse le fonctionnement de l'ironie chez Cioran en faisant une comparaison à Baudelaire et Kierkegaard :

On est « ironique » envers soi ou envers autrui en vue de dissoudre une certaine naïveté ou une certaine vulgarité spirituelle, de renverser, en l'humiliant, un certain désir de confort trop humain. On utilise donc un instrument tout à fait ascétique ; car telle est la fin de toute ascèse : macérer la chair, dissoudre les états de conscience nourris par le bien-être de cette chair'. (2009, p. 209)

8. Antoine Compagnon trace un mouvement similaire en ce qui concerne le doute chez Cioran : «Cioran insiste sur le doute du doute, le douteux du doute qui, sans dénivellation spéculative et par-delà l'affirmation, le maintiendrait dans l'ambiguïté. La sauvegarde du doute en tant que doute, c'est son défaut même, en substance, qu'il soit impossible» (1980, p. 462-3).

9. Voir aussi l'ouvrage de Patrice Bollon (1997) qui, sur l'ironie chez Cioran, constate : " Façon de travestir en connaissance de cause la réalité, l'ironie permet ainsi en société de réconcilier l'inconciliable : le devoir de vérité et la nécessité de l'illusion. Subtile chorégraphie du Vrai et du Faux, elle se présente en résumé comme l'instrument parfait d'un scepticisme accompli, adulte, serein, en ce qu'elle autorise à tirer de l'impératif du doute toutes ses conséquences, sans tomber pour autant dans l'impasse radicale [à laquelle] conduirait une application par trop rigide, mécanique, de ses principes » (p. 243). 
Eliade voit en Cioran un esprit ascétique dont un des buts serait de "réduire l'homme à ce qui lui est propre, [...] à ce qui ne dépasse pas la condition humaine : la vanité, les vers, la poussière » $(2009$, p. 209). Et c'est dans la négation que Michel Jarrety a localisé une certaine joie que la lecture des livres de Cioran peut susciter :

La négation est alors aussi bien réappropriation de ce qu'elle conteste, manière de vivre avec ce qu'on refuse, signe enfin d'une vigueur qui est la vie même.

C'est donc à tort qu'on l'interprèterait simplement comme le signe du malheur, la postulation du refus ou de l'affirmation de l'insoumission car une part essentielle de son bénéfice, au contraire, s'affirme dans le bonheur de nier qui seul permet de supporter le réel tel qu'il est : d'où la tonicité si souvent remarquée qui rend parfois étrangement gaie la lecture de Cioran et procède directement de la jubilation sensible, et justement de la dérision, qui accompagnent les modalités de la négation que son écriture multiplie. (1999, p. 130)

Si tout est vain, y inclus l'affirmation de la vanité, y inclus la tentative de mettre fin à la vanité en mettant fin à sa vie, ironiquement la vanité ne devrait pas nous dissuader de continuer à vivre. Puisqu'il est impossible d'imaginer une vie où tout ne serait pas vain, force nous est de vivre le mieux possible dans celle-ci. La résignation est parfois la voie préférée :

J'accepte la vie par politesse : la révolte perpétuelle est de mauvais goût comme le sublime de suicide. À vingt ans on fulmine contre les cieux et l'ordure qu'ils couvrent ; puis on s'en lasse. La pose tragique se sied qu'à une puberté prolongée et ridicule; mais il faut mille épreuves pour en arriver à l'histrionisme du détachement. (Cioran, 2011, p. 101)

Oublier la vanité en permanence nous jetterait dans l'illusion, mais un rappel constant ne fait rien pour annuler la vanité. Reste alors l'acte de penser contre soi, de tourner tout en négation, y inclus la négation elle-même. Paradoxalement, c'est la vanité qui sert à ouvrir la porte à cette double négation tout en nous mettant en garde contre l'illusion ou, disons-le franchement, le danger de l'optimisme.

\section{RÉFÉRENCES}

Acquisto, J. (2015). The Fall Out of Redemption: Writing and Thinking Beyond Salvation in Baudelaire, Cioran, Fondane, Agamben, and Nancy. New York, NY : Bloomsbury.

Bollon, P. (1997). Cioran, l'hérétique. Paris, France : Gallimard.

Cavaillès, N. (2011). Cioran malgré lui. Paris, France : Éditions CNRS.

Cioran, E. (1997). Cahiers. Paris, France : Gallimard.

Cioran, E. (2011). CEuvres. Paris, France : Gallimard.

Compagnon, A. (1980). Éloge des Sirènes. Critique, 396, 457-473.

David, S. (2006). Cioran. Un héroïsme à rebours. Montréal, Québec: Les Presses del'Université de Montréal. 
Eliade, M. (2009). Ascèse. Dans L. Tacou (dir.), Les Cahiers de l'Herne : Cioran (p. 209-210). Paris, France : L'Herne.

Jarrety, M. (1999). La morale dans l'écriture : Camus, Char, Cioran. Paris, France : PUF.

Liiceanu, G. (1995). Itinéraires d'une vie : EM Cioran, suivi de Les Continents de l'insomnie, entretien avec EM Cioran, traduit du roumain par Alexandra Laignel-Lavastine. Paris, France : Éditions Michalon.

Mauriac, C. (2009). L’un des meilleurs écrivains français. Dans L. Tacou (dir.), Les Cahiers de l'Herne : Cioran (p. 215-218). Paris, France : L'Herne.

Nadeau, M. (2009). Un penseur crépusculaire. Dans L. Tacou (dir.), Les Cahiers de l'Herne : Cioran (p. 211-212). Paris, France : L'Herne.

Stanisor, M.-G. (2015). Aveux et anathèmes ou la transformation de la philosophie. Dans A. Demars, N. Cavaillès, C. Lauren et M.-G. Stanisor (dir.), Cioran, archives paradoxales : Nouvelles approches critiques (p. 47-54). Paris, France : Classiques Garnier.

Zarifopol-Johnston, I. (2009). Searching for Cioran. Bloomington, IN : Indiana University Press.

RÉSUMÉ : Selon Emil Cioran, tout être humain est « imbu de la conviction que tout est vain ». Par contre, Cioran se demande tout de suite après avoir indiqué cette reconnaissance universelle de la vanité : "Mais qui ose en affronter les suites?». En quoi l'écrivain cioranien s'associe-t-il à cette humanité généralisée et en quoi est-ce qu'il s'en distingue?

Quelle doit être notre réaction alors, face à la vanité de tout ? On pourrait être tenté de dire que la destruction, et surtout l'autodestruction, semble être la réaction la plus raisonnée. Bien que Cioran écrive de manière quasi-obsessionnelle sur le thème du suicide, il n'arrive pas à le recommander comme solution à la vanité justement parce que la vanité résiste à toute solution. L'écrivain se voit ainsi dans une position contradictoire, car à quoi sert-il de déclarer la vanité de tout si cette déclaration ne fait que participer à cette même vanité ? L'acte de publier des livres n'est pas, selon Cioran, plus efficace que le suicide en ce qui concerne l'atténuation de la vanité, mais l'écriture et la lecture en tant que création et destruction simultanées nous permettent d'oublier la vanité au moins assez pour continuer à vivre.

Mots-clés : Cioran, vanité, suicide, écriture

\section{"But who dares to face the consequences ?": Emil Cioran and the Relations Among Vanity, Suicide, and Writing}

ABSTRACT: According to Emil Cioran, every human being is "full of the conviction that all is vain." But Cioran wonder immediately after indicating that universal recognition of vanity: "But who dares to face the consequences?" How does the writing voice in Cioran associate with and distinguish himself from this generalized humani- 
ty? And what should our reaction to the vanity of all things be? We could be tempted to say that destruction, and self-destruction above all, seems to be the most reasonable reaction. Although Cioran writes in a near-obsessional way about suicide, he does not ultimately recommend it as a solution to vanity precisely because vanity resists all solutions. The writer is thus in a contradictory position: what would be the use of declaring the vanity of all things if that declaration only participates in that same vanity? The act of publishing books is not, according to Cioran, more effective than suicide as far as the attenuation of vanity is concerned, but reading and writing as simultaneous creation and destruction allow us to forget vanity at least enough to continue living.

Keywords: Cioran, vanity, suicide, writing 